\title{
MODELAGEM DE DADOS PARA ANÁLISE DE EFICIÊNCIA NOS PARÂMETROS DE PERFURAÇÃO OFFSHORE
}

\author{
Yanko Sarzedas da Costa \\ Universidade Federal Fluminense \\ R. Passo da Pátria, 156, Niterói, RJ - CEP - 24210-240, Brasil \\ ys costa@id.uff.br \\ Professor Dr. Gilson Brito Alves Lima \\ Universidade Federal Fluminense \\ R. Passo da Pátria, 156, Niterói, RJ - CEP - 24210-240, Brasil \\ glima@id.uff.br \\ Professora Dra. Fabiana Rodrigues Leta \\ Universidade Federal Fluminense \\ R. Passo da Pátria, 156, Niterói, RJ - CEP - 24210-240, Brasil \\ fabianaleta@id.uff.br

\section{Dr. Hardy Leonardo da Cunha Pereira Pinto} \\ Petrobras - CENPES \\ Avenida Horácio Macedo, 950, Cidade Universitária, Rio de Janeiro - RJ - CEP 21941-915, \\ Brasil \\ hardy@petrobras.com.br

\section{Dr. Claudio Benevenuto de Campos Lima} \\ Petrobras \\ Av. Henrique Valadares, 28 - Centro, Rio de Janeiro - RJ - CEP 20231-030, Brasil \\ clima@petrobras.com.br
}

\section{RESUMO}

A indústria de petróleo busca a redução de custo e a melhoria da produtividade com o uso de tecnologia, métodos estatísticos e de apoio a decisão. Isso se deve ao fato das variações do mercado de petróleo e questões político-econômicas que levaram a redução dos hidrocarbonetos. Esse artigo foca na análise de eficiência de perfuração de poços pelo algoritmo Data Envelopment Analysis (DEA), sendo os dados selecionados com os métodos de Análise de Variância conferidos pelo Teste de Tukey. O resultado alcançado foi assegurar quais perfurações de poços foram apontados como eficiente para se tornarem modelos de planejamentos futuros.

Palavra-chave: DEA; ANOVA; Perfuração; Petróleo; Tukey; Taxa de Aprofundamento. 


\begin{abstract}
The oil industry seeks to reduce costs and improve productivity through the technology, statistical and decision support methods. This is due to variations in the oil market and political and economic issues that led to the hydrocarbon's reduction. This paper focuses on the well drilling efficiency analysis using the Data Envelopment Analysis (DEA) algorithm, and the data collected and selected by analysis of variance and Tukey test. The result was to ensure that well drilling has been identified as efficient to become future planning models.
\end{abstract}

Keywords: DEA; ANOVA; Drilling; Oil; Tukey; Rate of Penetration; ROP.

\title{
Como Citar:
}

COSTA, Yanko Sarzedas da et al. Modelagem de dados para análise de eficiência nos parâmetros de perfuração Offshore. In: SIMPÓSIO DE PESQUISA OPERACIONAL E LOGÍSTICA DA MARINHA, 19., 2019, Rio de Janeiro, RJ. Anais [...]. Rio de Janeiro: Centro de Análises de Sistemas Navais, 2019.

\section{INTRODUÇÃO}

Dividida em diferentes blocos e campos de exploração, a região do Pré-sal tornouse uma importante reserva de recursos para o País. Entretanto, com a queda do preço do barril do petróleo, devida a questões político-econômicas, a extração do hidrocarboneto teve suas atividades reduzidas nos últimos anos. Uma das principais considerações é que o breakeven dos projetos de exploração em águas ultra profundas no Brasil (Pré-sal) está em média acima dos 60 dólares o barril [1]. Isto é, existiam alguns projetos de desenvolvimento de poços, que eram considerados viáveis nas condições econômicas vigentes em 2014 (preço médio do barril de 101 dólares) e deixaram de ser no novo contexto de 2018 (preço médio do barril de 55 dólares).

A indústria de petróleo, com o intuito de melhorar a produtividade e reduzir custos, tem buscado o uso de tecnologias digitais para mitigar ou combater os efeitos das sazonalidades dos preços do petróleo e a escassez de profissionais. Além disto, os líderes da indústria apontam a necessidade de mudanças radicais no modelo operacional da indústria, sustentadas por uma maior adoção de tecnologias disruptivas. Corroborando com esta ideia, o relatório sobre a transformação digital do Fórum Econômico Mundial identifica Big Data, Data Analytics, Internet of Things, Mobility, Cloud e Artificial Intelligence como as principais prioridades para o investimento em tecnologia no setor de petróleo e gás [2].

A província do Pré-sal representa para o Brasil uma nova fronteira exploratória, onde o desafio de produzir óleo e gás está no fato de ser um ambiente marinho de águas ultra profundas, que alcança 3000 metros de coluna d’água e possuir uma camada de sal com espessura superior a 2000 metros de profundidade vertical, com reservatórios entre 5000 e 7000 metros de profundidade medida, conforme figura 1. 


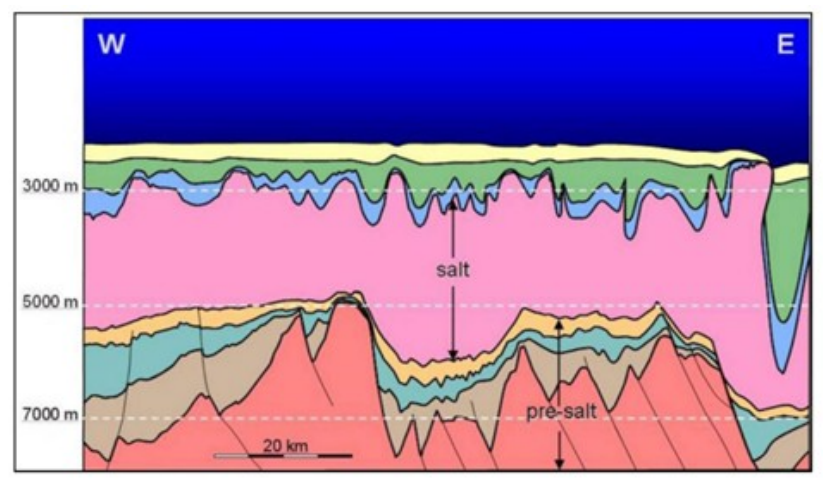

Figura 1: Intervalo Salino

[3]

Neste contexto, busca-se analisar, através das técnicas de mineração de dados, a eficiência do processo de perfuração dos poços de petróleo. Os dados desta pesquisa foram trabalhados através de linguagem de programação e mensurados com a metodologia de Análise Envoltória de Dados (DEA-Data Envelopment Analysis), através de um dos seus modelos clássicos DEA-BCC. As origens do DEA foram desenvolvidas inicialmente por [4], que considera retornos constantes de escala, e posteriormente um modelo complementar foi desenvolvido por [5], o BCC que considera retornos variáveis de escala.

Os resultados analisados permitiram identificar as unidades de perfuração, que foram referências de eficiência na utilização de parâmetros de perfuração em um determinado trecho de formação rochosa, que não terão sua litologia apresentada por questões de confidencialidade.

Dentro da finalidade desse artigo, serão analisados os parâmetros de perfuração, revolução, carregamento sobre broca e taxa de aprofundamento quanto a similaridades dos dados gerados durante uma operação de perfuração. A busca dessas similaridades será através da análise de variância (ANOVA) [6], a fim de selecionar poços que possam ser comparados através do método DEA no modelo BCC-O.

Neste sentido, o artigo encontra-se organizado em 5 seções. A primeira seção apresenta o contexto da pesquisa; a segunda explana os parâmetros de perfuração considerados para medir a eficiência entre poços; a seção seguinte aborda o método de avaliação de eficiência; a quarta seção refere-se à modelagem dos resultados, a partir da aplicação dos métodos ANOVA e DEA-BCC-O; na quinta e última seção, são discutidos os resultados e as considerações finais.

\section{PARÂMETROS DE PERFURAÇÃO}

Na fase do planejamento de perfuração do poço, parâmetros de referência são estabelecidos a partir de um programa de perfuração, feito por especialistas que possuem experiência de campo na área em que o poço será desenvolvido. Na realização de qualquer perfuração, segundo [7], esses parâmetros são acompanhados, controlados pelo sondador e estão relacionados, principalmente, ao carregamento aplicado sobre broca e velocidade da revolução da broca.

Segundo [8], as principais funções da coluna de perfuração são as seguintes: transmitir a revolução da mesa rotativa até a broca e produzir carregamento sobre broca, de forma que a perfuração seja efetiva e entregue a maior taxa de aprofundamento possível, 
desde que dentro dos limites operacionais vigentes.

Na perfuração, através do método rotativo, a broca consegue triturar a rocha e avançar em direção ao seu objetivo geológico. Nesse método, os cascalhos, gerados pela perfuração, são removidos do poço e carregados à superfície pelo fluido de perfuração. Este fluido é bombeado por dentro da coluna de perfuração e retorna pelo espaço existente entre a coluna e as paredes do poço [9].

Nesse processo de perfuração, equipamentos especializados em medição geram dados dos parâmetros de perfuração. [10] descreve a possibilidade de problemas de perfuração, como turbilhão, vibrações e oscilações que devem ser captados e analisados em tempo real para mitigação de problemas. O sistema proposto por [10] analisa dados de aceleração linear tri-axial, aceleração angular, peso sobre a broca (WOB), torque sobre a broca (TOB), rotação por minuto (RPM), campo magnético tri-axial, pressão interna e externa, para fazer recomendações ao operador de perfuração.

Na área de perfuração de poços de petróleo há aplicação de técnicas da ciência de dados. [11], em seu trabalho, usa aprendizagem de máquina para prever a taxa de perfuração (ROP) baseados no WOB e no RPM.

Apresentaremos a seguir os parâmetros utilizados ao modelo DEA-BCC em estudo.

- Carregamento sobre broca

O carregamento sobre broca aplicado na perfuração é proveniente da coluna de perfuração. Esta é composta por diferentes tubos conectados a ferramentas de aquisição de dados de superfície e direcionamento de poço, que tem por função realizar a perfuração e direcionar o poço até o seu objetivo geológico [12]. Toda essa coluna de equipamentos conectados a um sistema rotativo de perfuração proporciona uma carga que é suspensa por cabos. Esta carga é responsável por transferir carregamento para a broca. À medida que o operador suspende o sistema rotativo, o carregamento sobre a broca é aliviado. Em contrapartida, quando este libera a coluna, o carregamento é transferido para o fundo do poço.

A quantidade de carregamento transferida para a broca é determinada por especificações técnicas dos equipamentos que devem respeitar seus limites mecânicos, a fim de não acarretar futuros problemas na perfuração, como o desgaste precoce da broca, ou a flambagem dos tubos de perfuração, conforme [13]. Análises de esforços mecânicos, como limite de escoamento, dentre outros, são efetuadas previamente por softwares específicos de perfuração para gerar uma recomendação de trabalho, a partir dos limites e margens de segurança estipuladas.

- Revolução da broca

A importância da velocidade de giro da coluna é a transferência de revolução à broca de perfuração que, através de seus cortadores, irá cortar a formação e remover partes das rochas, que serão carregadas para a superfície através do fluido. Segundo [14], a revolução da broca é um parâmetro que controla também a frequência de contato do BHA com a parede do poço, que não é desejável pelos operadores de campo por gerar redução no desempenho do corte da formação, refletindo nas taxas e resultados da perfuração.

Os valores de revolução aplicados à coluna de perfuração são previamente 
analisados a partir do limite operacional dos equipamentos da sonda, responsáveis pelo giro da coluna, e pelas especificações técnicas da broca [15]. O número de revoluções acumuladas na broca durante a perfuração afeta diretamente o desgaste da superfície cortante, fato que pode levar ao comprometimento do diâmetro inicial da broca e à queda na taxa de aprofundamento.

- Taxa de aprofundamento

A taxa de metros perfurados por hora é uma medida largamente utilizada na indústria do petróleo para monitorar a velocidade do avanço em que a coluna de perfuração se movimenta em direção ao objetivo geológico ou reservatório, a ser explorado. A otimização da taxa de aprofundamento é entendida através de sua maximização, uma vez que sua redução implica em maior custo para a operação [16].

Este desempenho pode variar em função de diversos fatores, dentre eles o tipo da formação e os parâmetros utilizados na operação. Cada tipo de geologia possui características litológicas distintas, como, por exemplo, sua dureza e porosidade, que influem diretamente na velocidade de avanço da broca. Neste trabalho, as análises serão concentradas apenas nas formações derivadas do sal, que, por possuírem determinadas propriedades, dificultam o avanço da perfuração.

O tipo de formação que será perfurada é um fator independente da vontade humana. No entanto, os parâmetros de perfuração podem ser conduzidos pelos especialistas para identificar a melhor combinação entre carregamento e revolução na broca, que proporcionam a melhor taxa, conforme ilustra a figura 2. Entretanto, é interessante lembrar que as taxas de penetração não são proporcionais à maior ou menor aplicação dos parâmetros de entrada e sim ao ajuste dos parâmetros às condições da rocha ou até mesmo da dinâmica da perfuração, que podem necessitar de mudanças mediante às suas dificuldades de avanço.

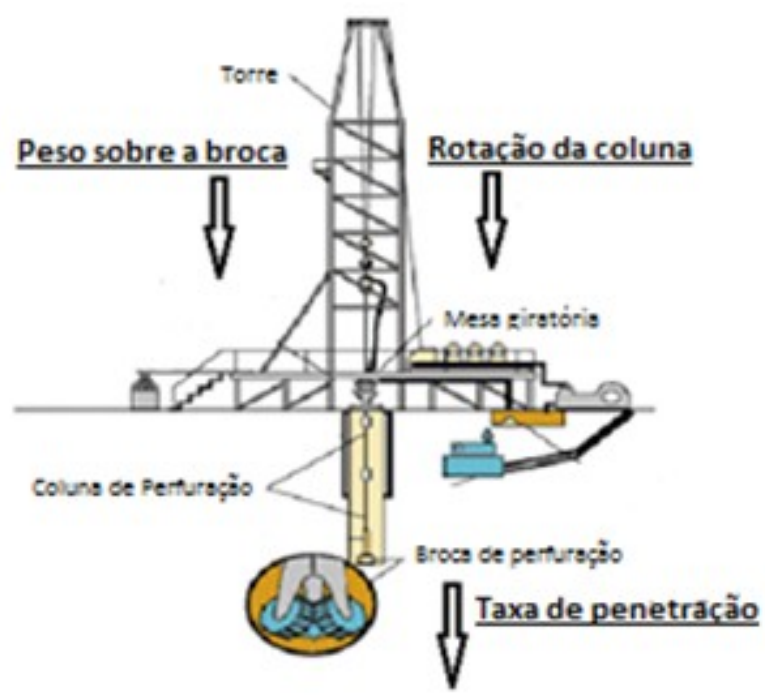

Figura 2: Vetores de Perfuração

[17] 


\section{AVALIAÇÃO DE EFICIÊNCIA}

A análise envoltória de dados tem como principal objetivo calcular a eficiência de unidades produtivas, chamadas de unidades de tomada de decisão ou DMUs (Decision Making Units), conhecendo-se os níveis de recursos utilizados e de resultados obtidos. Em sua formulação, usa problemas de programação linear (PPLs), que otimizam cada observação individual, de modo a estimar uma fronteira eficiente (linear por partes), composta das unidades que apresentam as melhores práticas dentro da amostra em avaliação. Essas unidades servem como referência ou benchmark para as unidades ineficientes [18].

A vantagem do DEA frente a outros modelos de produção é a capacidade de incorporar múltiplos inputs (entradas, recursos, insumos ou fatores de produção) e múltiplos outputs (saídas ou produtos) para o cálculo de uma medida de eficiência única, com ou sem a incorporação de julgamentos subjetivos por parte dos decisores.

A comparação entre as distintas produtividades faz referência ao conceito de eficiência das unidades produtivas, conforme figura 3 , ou seja, o que faz determinada unidade produzir em maior quantidade do que a outra, utilizando a mesma quantidade de recursos, ou consumindo menos que a anterior obtendo a mesma quantidade de produtos [19].

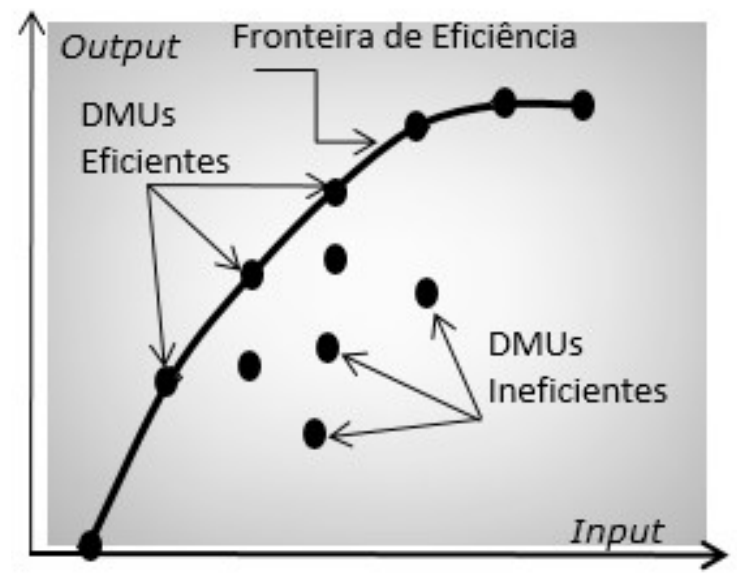

Figura 3: Esquema Gráfico DEA

[17]

Os modelos clássicos em DEA, mais amplamente utilizados são: o CCR e o BCC. O primeiro tem sua sigla oriunda do nome dos autores Charnes, A.; Cooper, W. W.; Rhodes (1978), que publicaram o primeiro artigo sobre o DEA. O segundo é uma extensão do primeiro, como se verá adiante e tem sua sigla oriunda do nome dos autores Banker, R. D.; Charnes, A.; Cooper (1984).

Para [20], o modelo CCR admite retornos constantes de escala (Constant Returns to Scale-CRS) que procuram maximizar a quantidade de produtos sujeitos à combinação viável de recursos utilizados. No entanto, nem todas as organizações trabalham com retornos constantes de escala. Nestas condições, o modelo CCR pode gerar medidas de eficiência mascaradas pela escala [21].

\subsection{Modelo BCC ORIEnTAdo a SAÍdA}

O cálculo da eficiência foi orientado a saída baseado nos parâmetros préestabelecidos pela engenharia de perfuração no programa de poço entregue aos operadores 
antes de cada operação. Portanto, a partir desses valores, pretende-se encontrar a combinação mais eficiente entre carregamento sobre broca e revolução da broca para entrega de taxa em metros perfurados por hora, relação obtida com retornos variáveis de escala, uma vez que os parâmetros de entrada no sistema não possuem uma proporcionalidade direta com os valores de saída.

Analisando as possibilidades da metodologia DEA, o modelo mais indicado para esta análise foi o modelo BCC-O (orientado para maximização dos outputs), devido aos seguintes fatores:

- A taxa de aprofundamento obtida não guarda proporcionalidade com os parâmetros de entrada no sistema, reforçando o conceito do modelo BCC (retornos variáveis de escala).

- Os insumos não podem ser otimizados em função da taxa de aprofundamento, uma vez que são pré-determinados pelos programas de engenharia de poço. Isso reforça a orientação ao output que deve ser maximizado em função de reduzir elevados custos de exploração, como diárias de plataformas e equipamentos a bordo.

Para que seja a melhor possível em relação as outras, cada DMU escolhe seus carregamentos, de modo a corroborar com sua eficiência. Entretanto, isso pode colocar outras DMUs na fronteira de eficiência, provocando muitas unidades com 100\%, reduzindo a discriminação entre elas, conforme o modelo Dual (Multiplicadores) exposto na formulação a seguir.

$$
\begin{gathered}
\min h_{0}=\sum_{i=1}^{r} v_{i} x_{i 0}-v_{*} \\
\text { Sujeito } a: \\
\sum_{j=1}^{s} u_{j} y_{j k}=1, K=1,2, \ldots, n \\
\sum_{i=1}^{r} v_{i} x_{i k}-\sum_{j=1}^{s} u_{j} y_{j k}-v_{*} \leq 0, K=1,2, \ldots, n \\
u_{j} \text { e } v_{i} \geq 0 \forall j, i \\
v_{*} \in \mathbb{R}
\end{gathered}
$$

onde:

$\mathrm{h}_{0}$ é a Eficiência

$\mathrm{x}_{i k}, y_{j k}=$ Inputs $i$ e Outputs $j$ da DMU

$\mathrm{x}_{i 0}, y_{j 0}=$ Inputs $i$ e Outputs $j$ da DMU

\subsection{FRONTEIRA INVERTIDA}

Visando aprimorar o poder de discriminação do modelo utilizado, pode-se utilizar a 
técnica da fronteira invertida, que tem por base considerar o que antes era input como sendo output e vice-versa. Desta forma, é feita uma análise de eficiência do ponto de vista pessimista.

Para ordenação final levando em consideração a fronteira invertida, segundo [19], é construído um índice de eficiência composta $(E f f c)$. Ele é definido como sendo a média aritmética entre a eficiência em relação à fronteira DEA convencional e o complemento da eficiência em relação à fronteira invertida, conforme apresentado na equação [6]. Essa técnica melhora na discriminação das DMUs eficientes sem incluir julgamento a priori. Para uma DMU ter um bom índice composto é avaliada com um bom desempenho sem ter nenhum desempenho fraco, não permitindo a especialização das DMUs.

$$
E f f c=\frac{E f f p+(1-E f f i)}{2}
$$

onde:

E f f $c$ - Eficiência composta

E f f $p$ - Eficiência padrão

E f $f i$ - Eficiência invertida

\section{MODELAGEM E RESULTADOS}

\subsection{DEFINIÇÃO DAS ENTRADAS E SAÍDAS}

O procedimento da análise de variância dos parâmetros revolução e carregamento sobre a broca entre poços visa a seleção de poços que apresentam uniformidades operacionais. Com isso, os parâmetros revolução e carregamento sobre broca serão submetidos ao DEA como inputs e a taxa de aprofundamento será submetido como output.

Como resultado, é obtida a taxa de eficiência da operação de perfuração de cada poço. Os poços apontados como eficientes poderão ter seus parâmetros indicados como modelos em planejamentos de futuras perfurações de poços.

\subsection{PROCESSO DE SELEÇÃO DOS POÇOS}

Existem diversos desafios científicos no que tange à compreensão do comportamento do poço, bem como à heterogeneidade das rochas e a melhor estratégia para a exploração de hidrocarbonetos. Além disso, existe também para [22] a necessidade de capacitação de mão-de-obra qualificada frente ao desenvolvimento de novas estratégias e maquinários na exploração e desenvolvimento de poços.

A escolha dos parâmetros de entrada na perfuração de poços provoca diferentes resultados quando se analisa a taxa de aprofundamento como resultado esperado, que, na maioria dos casos, deve ser sempre a maior possível, exceto quando há alguma necessidade de evitar o acúmulo de cascalho no poço. Portanto, a partir dos dados obtidos, a pesquisa busca comparar e analisar a eficiência de poços relacionados a um mesmo perfil geológico de perfuração, com o objetivo de evidenciar melhores ajustes de parâmetros relacionados a seu respectivo desempenho.

A avaliação dos parâmetros de perfuração, segundo [23], ainda hoje é feita pela sensibilidade e experiência do operador de campo, fato este que torna a busca por melhores resultados suscetíveis à falha. Neste sentido, torna-se interessante o estudo e aplicação de 
ferramentas para avaliação de eficiência do evento, com base em fatores quantitativos, reduzindo assim a subjetividade da análise.

No procedimento adotado, serão avaliados os dados dos parâmetros de perfuração exógenos, selecionados ao modelo DEA para averiguação de eficiência em perfuração: revolução da broca e o carregamento aplicado sobre a broca. Os dados são referentes a 6 poços perfurados em seus respectivos 4 campos petrolíferos. Com essa diversidade de dados, é necessário lançar mão da análise de variância (ANOVA), com o objetivo de selecionar os poços com o mesmo perfil de revolução e carregamento sobre a broca. Uma vez estabelecido o conjunto de poços, estes devem ser submetidos ao algoritmo DEA, a fim de obter o poço com melhor eficiência em taxa de aprofundamento em relação à revolução e ao carregamento aplicado. $\mathrm{O}$ poço com maior taxa de eficiência poderá servir como referência no planejamento de futuros projetos de perfuração.

Serão estabelecidos os dados de revolução e carregamento sobre a broca para a verificação da uniformidade das médias dos dois parâmetros, através do método ANOVA. Então, as inferências sobres as hipóteses 7 e 8 são especificadas para os correspondentes parâmetros:

$$
\begin{gathered}
H_{0}: \mu_{1}=\mu_{2}=\mu_{4}=\mu_{6}=\mu_{7}=\mu_{8} \\
H_{a}: \text { As médias são diferentes }
\end{gathered}
$$

A tabela 1 refere-se às médias das rotações a cada 100 metros, consideradas a partir da profundidade inicial (PI) para inferir sobre as hipóteses 7 e 8.

Tabela 1: Tratamento da revolução nos poços a cada 100 metros.

\begin{tabular}{c||cccccc}
\hline Profundidade & Poço-01 & Poço-02 & Poço-04 & Poço-06 & Poço-07 & Poço-08 \\
\hline \hline PI & 190,3288 & 196,1962 & 201,1261 & 198,3510 & 175,5011 & 160,0745 \\
\hline PI + 100 & 188,7887 & 190,5556 & 201,2826 & 198,6849 & 182,7025 & 161,0087 \\
\hline PI + 200 & 184,9615 & 195,5028 & 200,9878 & 194,5411 & 179,0195 & 161,1235 \\
\hline PI + 300 & 181,4097 & 195,0214 & 200,0920 & 194,9711 & 193,2769 & 160,7822 \\
\hline PI + 400 & 174,0690 & 159,8458 & 200,5580 & 185,0242 & 195,0917 & 161,1970 \\
\hline PI + 500 & 168,4178 & 168,0429 & 201,5043 & 198,6333 & 195,1657 & 161,3420 \\
\hline PI + 600 & 165,9807 & 174,3264 & 199,1802 & 196,1287 & 197,0574 & 160,8050 \\
\hline PI + 700 & 186,9004 & 173,9235 & 199,6852 & 193,3632 & 190,7917 & 160,3096 \\
\hline PI + 800 & 183,6891 & 168,9962 & 200,7644 & 195,8726 & 189,8174 & 164,7528 \\
\hline PI + 900 & 184,7569 & 161,7021 & 201,3343 & 139,8595 & 178,8505 & 177,5063 \\
\hline \hline Médias & 180,9303 & 178,4113 & 200,6515 & 189,5430 & 187,7275 & 162,8902 \\
\hline
\end{tabular}

A tabela 2 refere-se às médias dos carregamentos sobre broca a cada 100 metros, a partir da profundidade inicial, para inferir sobre as hipóteses 7 e 8.

Tabela 2: Tratamento do carregamento nos poços a cada 100 metros. 


\begin{tabular}{c||cccccc}
\hline Profundidade & Poço-01 & Poço-02 & Poço-04 & Poço-06 & Poço-07 & Poço-08 \\
\hline \hline PI & 22,8127 & 26,8641 & 41,6348 & 39,6523 & 46,5241 & 44,4782 \\
\hline PI + 100 & 34,1263 & 33,0508 & 37,0817 & 34,0359 & 44,4601 & 42,4357 \\
\hline PI + 200 & 44,6065 & 37,3162 & 41,6783 & 29,2555 & 45,7119 & 38,7334 \\
\hline PI + 300 & 45,1731 & 37,7910 & 54,0175 & 34,2868 & 40,1611 & 43,0686 \\
\hline PI + 400 & 43,8377 & 28,0356 & 38,4380 & 27,7229 & 47,8105 & 43,5156 \\
\hline PI + 500 & 33,6130 & 30,2605 & 45,5339 & 34,6973 & 48,0195 & 44,0355 \\
\hline PI + 600 & 12,6648 & 39,5807 & 46,9970 & 27,7119 & 48,6743 & 45,4649 \\
\hline PI + 700 & 12,4955 & 38,1240 & 50,9640 & 22,1502 & 50,0190 & 43,4951 \\
\hline PI + 800 & 08,5315 & 33,8052 & 44,1561 & 24,0709 & 34,2994 & 34,0969 \\
\hline PI + 900 & 08,7581 & 29,8875 & 54,1194 & 17,1318 & 28,1108 & 38,8263 \\
\hline \hline Médias & 26,6619 & 33,4716 & 45,4621 & 29,0716 & 43,3791 & 41,8150 \\
\hline
\end{tabular}

Como pode ser observado na tabela 3 , o valor p referente a revolução e o carregamento tiveram as hipóteses nulas rejeitadas para o nível de significância $\alpha=0,05$, conforme os testes de hipóteses da ANOVA [6]. 
Tabela 3: Testes das Hipóteses.

\begin{tabular}{c||ccc}
\hline Hipótese & valor $\mathrm{p}$ & $\alpha$ & valor $\mathrm{p} \leq \alpha$ \\
\hline \hline $\mathrm{H}_{0}$ : Uniformidade da revolução & $9,75685 \mathrm{E}-09$ & 0,05 & Rejeita $\mathrm{H}_{0}$ \\
\hline $\mathrm{H}_{0}$ : Uniformidade do carregamento & $1,64934 \mathrm{E}-06$ & 0,05 & Rejeita $\mathrm{H}_{0}$ \\
\hline
\end{tabular}

Com isso, para a próxima etapa será aplicado o método de comparações múltiplas (MCM), conhecido como teste de Tukey [24]. Em geral, os MCMs buscam controlar o aumento da probabilidade do erro Tipo I ou diminuição do intervalo de confiança global, protegendo a taxa de erro da família dos testes (Familywise Error Rate (FWER)).

São apontados os contrastes entre as médias das famílias das rotações e dos carregamentos sobre a broca entre poços, conforme expresso na equação 9.

$$
\Gamma=\left|\mu_{i}-\mu_{j}\right|, \forall i \neq j
$$

O teste de Tukey consiste em definir a menor diferença significativa a partir da equação 10, sendo q um valor tabelado, QME o erro dos desvios da ANOVA e n o número de réplicas nos tratamentos, representados pelas médias das rotações e carregamentos para cada poço nos modelos observados nas tabelas 1 e 2 .

$$
T S D=q \cdot \sqrt{\frac{Q M E}{n}}
$$

Com o TSD calculado, encontra-se, para a família das rotações, o valor 18,58382854 e para a família dos carregamentos obteve-se 14,15045533. Assim, aplicandose a equação 11, pode-se averiguar as médias com diferenças significativas destacadas nas tabelas 4 e 5.

$$
T S D \leq \Gamma, \forall i \neq j
$$

Por conseguinte, foi utilizado o teste de Tukey para desprezar os poços cuja médias destoam nas famílias de rotações e carregamentos.

Assim, inicia-se a seleção dos poços 01, 02, 06 e 07 na tabela 4, por serem menores que 18,58382854 (TSD das rotações).

Tabela 4: Comparação entre as médias da revolução.

\begin{tabular}{c||cccccc}
\hline & Poço-01 & Poço-02 & Poço-04 & Poço-06 & Poço-07 & Poço-08 \\
\hline \hline Poço-01 & 0 & & & & & \\
\hline Poço-02 & 02,5190 & 0 & & & & \\
\hline Poço-04 & 19,7212 & 22,2402 & 0 & & & \\
\hline Poço-06 & 08,6127 & 11,1317 & 11,1085 & 0 & & \\
\hline Poço-07 & 06,7972 & 09,3162 & 12,9240 & 01,8155 & 0 & \\
\hline Poço-08 & 18,0401 & 15,5211 & 37,7613 & 26,6528 & 24,8373 & 0 \\
\hline
\end{tabular}

Para a seleção com base no carregamento, conforme a tabela 5, o poço 07 foi eliminado por ser maior que 14,15045533 (TSD do carregamento) quando cruzados quando os poços 1 e 6. 
Tabela 5: Comparação entre as médias dos carregamentos sobre a Broca.

\begin{tabular}{c||cccccc}
\hline & Poço-01 & Poço-02 & Poço-04 & Poço-06 & Poço-07 & Poço-08 \\
\hline \hline Poço-01 & 0 & & & & & \\
\hline Poço-02 & 06,8096 & 0 & & & & \\
\hline Poço-04 & 18,8002 & 11,9905 & 0 & & & \\
\hline Poço-06 & 02,4096 & 04,4000 & 16,3905 & 0 & & \\
\hline Poço-07 & 16,7172 & 09,9075 & 02,0830 & 14,3075 & 0 & \\
\hline Poço-08 & 15,1531 & 08,3435 & 03,6470 & 12,7435 & 01,5641 & 0 \\
\hline
\end{tabular}

Será elaborado um benchmarking entre os poços 01, 02 e 06, aplicando o modelo BCC-O, ou seja, buscando a melhor taxa de aprofundamento em relação às médias da revolução e às médias dos carregamentos sobre broca na profundidade estudada.

Os PPLs do modelo DEA são compostos pelas médias da revolução e do carregamento sobre broca, conforme as tabelas 1 e 2 . A variável taxa de aprofundamento é formada pela média da profundidade medida em metros para cada poço durante a perfuração, expressos na tabela 6 .

Tabela 6: Média da taxa de aprofundamento em metros.

\begin{tabular}{c||cccccc}
\hline Profundidade & Poço-01 & Poço-02 & Poço-04 & Poço-06 & Poço-07 & Poço-08 \\
\hline \hline Médias & 35,4095 & 32,6501 & 36,1580 & 19,6212 & 28,2363 & 37,5504 \\
\hline
\end{tabular}

O processo benchmarking se dá pela solução dos PPLs (Dual - Multiplicadores) elaboradas abaixo para a comparação do desempenho dos poços selecionados.

$$
\begin{array}{r}
\min 35.4094 v_{1}-v . \\
\text { sujeito a, } \\
180.9302 u_{1}+26.6619 u_{2}=1.0 \\
-180.9302 u_{1}-26.6619 u_{2}+35.4094 v_{1}-v_{*} \leq 0 \\
-178.4112 u_{1}-33.4715 u_{2}+32.6500 v_{1}-v_{*} \leq 0 \\
-189.5429 u_{1}-29.0715 u_{2}+19.6211 v_{1}-v_{*} \leq 0
\end{array}
$$

$\min 32.6500 v_{1}-v$.

sujeito a,

$178.4112 u_{1}+33.4715 u_{2}=1.0$

$-180.9302 u_{1}-26.6619 u_{2}+35.4094 v_{1}-v_{\bullet} \leq 0$

$$
-178.4112 u_{1}-33.4715 u_{2}+32.6500 v_{1}-v_{\bullet} \leq 0
$$$$
-189.5429 u_{1}-29.0715 u_{2}+19.6211 v_{1}-v_{\bullet} \leq 0
$$

$\min 19.6211 v_{1}-v$.

sujeito a,

$189.5429 u_{1}+29.0715 u_{2}=1.0$

$-180.9302 u_{1}-26.6619 u_{2}+35.4094 v_{1}-v_{\bullet} \leq 0$

$$
\begin{aligned}
& -178.4112 u_{1}-33.4715 u_{2}+32.6500 v_{1}-v_{\bullet} \leq 0 \\
& -189.5429 u_{1}-29.0715 u_{2}+19.6211 v_{1}-v_{\bullet} \leq 0
\end{aligned}
$$


A solução dos PPLs levou à constituição dos benchmarks demonstrados na tabela 7. Isso irá orientar quais os poços servem de modelo para planejamento de futuras perfurações.

Tabela 7: Eficiência das perfurações.

\begin{tabular}{c||cccc}
\hline DMU & Revolução & Carregamento & Aprofundamento & Eficiência \\
\hline \hline Poço-01 & 180,9303 & 26,6619 & 35,4095 & 1,00 \\
\hline Poço-02 & 178,4113 & 33,4716 & 32,6501 & 1,00 \\
\hline Poço-06 & 189,5430 & 29,0716 & 19,6212 & 0,55 \\
\hline
\end{tabular}

Pode-se observar que os parâmetros de revolução e carregamento do poço-01 e poço-02 têm taxa de aprofundamento classificadas como eficientes. O Poço-06 tem uma taxa de aprofundamento que destoa dos demais poços com uma aplicação de revolução acima dos demais poços. Isso conota que fatores exógenos à operação estão interferindo na eficiência da perfuração. Dentre os principais fatores que influenciam os resultados da perfuração, destacamos as características litológicas da rocha. Esses fatores impõem ao operador uma maior aplicação de revolução e carregamento sobre a broca para aumentar a taxa de aprofundamento.

\section{CONCLUSÕES}

A densidade dos dados provenientes dos sensores das plataformas, em conjunto com sua diversidade, torna a aplicação de técnicas de mineração de dados pertinentes ao estudo de questões que buscam otimizar a performance ou reduzir custos de produção na exploração de poços offshore, através do melhor entendimento da questão relacionada à eficiência durante a aplicação de parâmetros operacionais em operações off-shore.

Após a redução de dimensionalidade dos sensores envolvidos na perfuração, que foi identificada através de pesquisa bibliográfica e com a ajuda de especialistas, o estudo buscou apresentar uma proposta de análise e avaliação da eficiência relacionada à perfuração de poços, a fim de apresentar os parâmetros de revolução e carregamento sobre a broca como referência na busca pela melhor taxa de penetração. Com isso, optou-se pelo DEA orientado ao output.

Como não há uma relação direta entre a revolução, carregamento e taxa de aprofundamento, isso torna a relação de retornos de escala variáveis, o que sugere o uso do modelo BCC do DEA. Neste sentido, é importante ressaltar a quebra de um paradigma na indústria do petróleo, que considera a aplicação de parâmetros mais agressivos relacionados diretamente a uma melhor taxa de entrega. Entretanto, é possível perceber, na relação dos dados disponibilizados, que o ajuste entre os parâmetros e as condições do poço ainda são os mais favoráveis para uma melhor taxa de aprofundamento.

Um outro aspecto abordado foi a seleção de poços com formação geológica semelhante. Pois, a busca da melhor taxa de aprofundamento deve ser na mesma condição geológica, o que permite observar as melhores aplicações da revolução conjugada com o carregamento sobre a broca. Porém, nesse estudo, os dados da litologia não estavam disponíveis, o que impossibilitou o agrupamento de poços com formação litológica similar. Desse modo, para a escolha dos poços, como unidades produtivas, adotou-se o procedimento pelo teste estatístico ANOVA para inferir a uniformidade das médias entre os parâmetros de perfuração.

Consequentemente, foi estipulado um processo de apoio à elaboração de planejamento de perfurações de poços de petróleo que apura as práticas das melhores taxas 
de perfuração. A comparação e evidência dos parâmetros é útil no sentido de servir como orientação na fase de planejamento de poços, ou seja, para que o responsável pela recomendação prévia dos valores de revolução na broca e carregamento na broca tenha uma referência do poço eleito como mais eficiente de acordo com a metodologia proposta.

Como sugestões de desdobramento da pesquisa, podem ser apontadas a avaliação de outras variáveis para medir a eficiência da perfuração de poços, o que possibilitaria uma visão mais singular e adaptada do desempenho da perfuração.

\section{REFERÊNCIAS BIBLIOGRÁFICAS}

[1] WOODMACKENZIE. Reserves and oil breakeven price data. 2016. Disponível em: $<$ https://www.woodmac.com/research/products/upstream/upstream-data-tool/>.

[2] KUMAR. World economic forum for digital technologies-opportunities in the ep sector.2017. Disponível em: <https://webevents.spe.org/products/digitaltechnologiesopportunities-in-the-ep-sectortab-producttaboverview==>:

[3] FERREIRA A. P. L. A.; CARVALHO, D. J. L. R. R. M. S. D. M. T. I. J. B. R. C. A. S. B. Automated decision support and expert collaboration avoid stuck pipe and improve drilling operations in off-shore brazil subsalt well. 2015.

[4] CHARNES, A. et al. Measuring the efficiency of decision-making units. European journal of operational research, Elsevier, v. 3, n. 4, p. 339-338, 1979.

[5] BANKER, R. D.; CHARNES, A.; COOPER, W. W. Some models for estimating technical and scale inefficiencies in data envelopment analysis. Management science, INFORMS, v. 30, n. 9, p. 1078-1092, 1984.

[6] ANDERSON DENNIS J. SWEENEY, T. A. W. D. R. Essentials of Statistics for Business Economics, Sixth Edition. [S.l.]: South-Western College Pub, 2010.

[7] CARPENTER, C. et al. Stuck-pipe prediction with automated real-time modeling and data analysis. Journal of Petroleum Technology, Society of Petroleum Engineers, v. 68, n. 06, p. 72-73, 2016.

[8] MITCHELL, R. F.; MISKA, S. Z. Fundamentals of drilling engineering. [S.l.]: Society of Petroleum Engineers, 2017.

[9] MELLO, E. V. Top drive: aplicações e experiências em sistemas de perfuração. Universidade Federal Fluminense, 2014.

[10] MANSURE, A. J. et al. Interpretation of diagnostics-while-drilling data. 2003.

[11] HEGDE, C.; GRAY, K. E. Use of machine learning and data analytics to increase drilling efficiency for nearby wells. Journal of Natural Gas Science and Engineering, Elsevier B.V., p. 327-335, 2017.

[12] MATHIAS, V. M. Coluna de Perfuração em Poços de Petróleo. Dissertação (B.S.thesis) - Universidade Federal do Rio Grande do Norte, 2016.

[13] BRAGA, A. M. B. Diagnósticos de problemas operacionais durante a perfuração de poços de petróleo. Tese (Doutorado) - PUC-Rio, 2011.

[14] HESS, J. et al. Pipe sticking prediction using LWD real-time measurements. In: Society of Petroleum Engineers. IADC/SPE Drilling Conference and Exhibition. [S.l.], 2016. 
[15] MONTEIRO, H. L. S. Análise de desempenho de diferentes leis de controle de vibrações torcionais em colunas de perfuração de poços de petróleo. Tese (Doutorado) - Universidade de São Paulo, 2012.

[16] MOHAMMADSALEHI, M.; MALEKZADEH, N. et al. Application of new hole cleaning optimization method within all ranges of hole inclinations. In: International Petroleum Technology Conference. [S.l.], 2011.

[17] SCHUWARTZ F.; DUNCAN, L. B. G. G. L. O. H. L. C. D. B. C. Modelagem de parâmetros operacionais para suporte à avaliação do processo de perfuração de poços off-shore. 2019.

[18] MELLO, J. C. C. B. S. et al. Análise de envoltória de dados no estudo da eficiência e dos benchmarks para companhias aéreas brasileiras. Pesquisa Operacional, SciELO Brasil, v. 23, n. 2, p. 325-345, 2003.

[19] MEZA, L. A. et al. Isyds-integrated system for decision support (siad-sistema integrado de apoio à decisão): a software package for data envelopment analysis model. Pesquisa Operacional, SciELO Brasil, v. 25, n. 3, p. 493-503, 2005.

[20] SOUZA, P. C. d.; SCATENA, J. H. G.; KEHRIG, R. T. Aplicação da análise envoltória de dados para avaliar a eficiência de hospitais do sus em mato grosso. Physis: Revista de Saúde Coletiva, SciELO Public Health, v. 26, p. 289-308, 2016.

[21] FORGIA, G. M. L.; COUTTOLENC, B. F. Desempenho hospitalar no brasil. São Paulo: Singular, 2009.

[22] LIMA, T. D. d. Desafios tecnológicos na exploração de petróleo em águas profundas: o sistema Petrobras de inovação. Universidade Estadual Paulista (UNESP), 2015.

[23] GANDELMAN, R.; MARTINS, A. Real-Time Drilling Data Analysis: Building Blocks for the Definition of a Problem Anticipation Methodology. IADC/SPE Drillng . .. , 2010. Disponível em:<http://www.onepetro.org/mslib/servlet/onepetropreview? id=SPE-128871-MS\&soc=SPEnnhttp://www.onepetro.org/mslib/servlet/ onepetropreview?id=SPE-128871-MS>.

[24] HSU, J. Multiple Comparisons: Theory and Methods (Guilford School Practitioner). [S.l.]: Chapman and Hall/CRC, 1996.Primeira referência. Primeira referência. Primeira referência. Primeira referência. Primeira referência. 